\section{ABSTRACT}

\title{
The Characteristics of free surface vortices at low-head hydropower intakes
}

\author{
Frank Suerich-Gulick ${ }^{1}$, Susan J. Gaskin ${ }^{2}$, Marc Villeneuve ${ }^{3}$, and Étienne Parkinson ${ }^{4}$
}

Tools for engineers who assess and optimize hydropower intakes are provided to help them measure and quantify the characteristics of free surface vortices (characteristic radius, bulk circulation, tip depth, nominal depression slope) that form at the intakes. Accessible methods are proposed for measuring and modelling vortex characteristics and the processes that affect their generation and strength. Common mechanisms that produce and strengthen the vortices (flow separation, shear, asymmetric approach flow) are discussed. An analytical model, based on Burgers's vortex model and laboratory measurements, is described that incorporates the effect of the approach flow and intake geometry on vortex characteristics. Simple measurement techniques (acoustic Doppler velocimetry and surface particle tracking velocimetry) are presented by which the flow and vortex characteristics can be documented, allowing the model to be adjusted to the particularities of the specific intake under consideration. The analytical model is then used to help understand how the different processes affect the scaling of vortex characteristics.

\footnotetext{
${ }^{1} \mathrm{PhD}$ Student, Dept. of Civil Engineering and Applied Mechanics, McGill University

${ }^{2}$ Associate Professor, Dept. of Civil Engineering and Applied Mechanics, McGill University, MacDonald Engrg Bldg., Rm. 492, 817 Sherbrooke St. W., Montréal, QC, H3A OC3, Canada. E-mail: susan.gaskin@mcgill.ca

${ }^{3}$ Groupe Conseil Lasalle

${ }^{4}$ Andritz Hydro
} 
Keywords: hydropower intakes, free surface vortex, physical scale modeling, scale effects, viscosity, surface tension, turbulence

\section{Introduction}

Vortices occur in a wide range of scales, in natural and man-made systems, in fluids such as air and water. They are a fundamental component of turbulence. Free surface vortices are sometimes observed near the intake of hydroelectric plants, with one end connected to the free surface and the other entering the inlet. Their occurrence is problematic because they can entrain air or debris or lead to unsteady or non-uniform flow at the turbines. Their impact ranges from simply reducing the power output of the plant to causing premature degradation of mechanical components. Free surface vortices occur most commonly at lower head run-of-river plants where they are most likely to be harmful due to the limited distance between the inlet and the turbines and to the often limited flow-aligning devices. There is great diversity in the layout of run-of-river plants; Fig. 1 shows sample schematic plan and side section views. Free surface vortices have on occasion been observed at high head plants with large reservoirs under very specific conditions.

This work aims to help practicing engineers assess and interpret vortex activity in physical scale models of intakes to help reduce the risk of problematic vortices forming in the full-scale, 'prototype' intake. We begin by discussing the processes that contribute to the generation of free surface vortices at intakes and present a relatively simple analytical model that was developed using measurements made in a simplified intake lab model. The model links vortex characteristics to the geometry and flow conditions and it is presented 
in such a manner that it can be adapted by the reader to other intakes using data that can be collected with relatively accessible measurement devices.

We discuss the implications of the analytical model in terms of vortex characteristics that are observable and relevant to intake designers and plant operators, such as the shape and depth of the free surface depression. Finally, the analytical model is used to estimate how the characteristics of a vortex observed in a laboratory model would appear in the prototype intake. Scale effects due to surface tension as well as viscosity are predicted in quantitative terms and the implications and limitations of these predictions are discussed.

\section{Mechanisms controlling vortex strength}

Although in practice vortices are complex and unsteady, much can be grasped about how they are generated and what determines their intensity from a relatively simple analytical vortex model. In this section, Burgers's vortex model is presented. A description of the different ways in which vorticity can be generated at an intake follows. The concentration of vorticity into a vortex of greater intensity by axial stretching is also discussed.

A vortex can be thought of as a local concentration of vorticity within which streamlines follow a circular, helical, or spiral pattern. Vorticity is a vector quantity defined mathematically as the curl of the velocity field: $\boldsymbol{\omega}=\boldsymbol{\nabla} \times \boldsymbol{V}$ (Saffman, 1992). In physical terms it describes the rotation of a local fluid particle about its center of mass. In viscous fluids, vorticity concentrated in the vortex core gets smeared outward by diffusion, producing a smooth radial profile that resembles a bell curve. In free surface intake vortices, the diffusion's spreading effect is counteracted by the concentrating effect of axial vortex 
stretching that is driven by the flow accelerating down towards the intake's inlet. Burgers's model describes a steady vortex produced by a state of equilibrium between these two processes.

\section{Burgers's vortex model}

Burgers's vortex model assumes that the flow is axisymmetric, that the radial velocity depends only on the radial distance $r$ from the vortex axis and that the axial velocity varies linearly and only as a function of the axial coordinate $z: V_{r}(r)=-a r / 2, V_{z}(z)=a z$ (Burgers, 1948). The gradient $a$, a constant with units of $\mathrm{s}^{-1}$, indicates the rate of axial stretching that the vortex is subjected to: $a=\partial V_{z} / \partial z$, where the $z$-axis is defined pointing downward from the free surface. The profile of $V_{r}$ is defined so that continuity is satisfied. Solving the axisymmetric Navier-Stokes equations with these prescribed velocity profiles produces an azimuthal velocity $V_{\theta}(r)$ field that depends only on the radial coordinate and is constant along the vortex axis $z$ :

$$
V_{\theta}(r)=\frac{\Gamma_{\infty}}{2 \pi r}\left(1-\exp \left(-\left(r / r_{o}\right)^{2}\right)\right)
$$

where $\Gamma_{\infty}$ is the bulk circulation of the vortex, and $r_{o}$ is the characteristic radius of the vortex. In Burgers's model, $r_{o}$ is controlled by the ratio of the molecular viscosity $\nu$ (units $\mathrm{m}^{2} / \mathrm{s}$ ) to the axial gradient $a$ :

$$
r_{o}=2(\nu / a)^{1 / 2}
$$

This relation shows that a stronger axial gradient $a$ causes the vortex to contract into a tighter vortex with a smaller characteristic radius, while increased viscosity causes the 
vortex to spread outward.

The bulk circulation can be obtained by integrating the axial vorticity $\omega_{z}$ across the entire vortex cross-sectional area $\mathbf{A}: \Gamma_{\infty}=\int_{\mathbf{A}} \omega_{z} d \mathbf{A}$, or by performing a line integral of the azimuthal velocity $V_{\theta}$ along the full circumference of the circle $C$ that encloses the vortex: $\Gamma_{\infty}=\oint_{\mathbf{C}} \mathbf{V} \cdot d \mathbf{C}$. The second approach is easiest to compute from experimental data since it is difficult to measure vorticity directly. Setting $r=4 r_{o}$ as the upper limit of the integration is sufficient to measure the bulk circulation within reasonable accuracy since the bulk of vorticity is concentrated within the vortex core $\left(r<r_{o}\right)$ and drops off to a negligible amount beyond $r>3 r_{o}$.

Burgers's model captures the flow inside the vortex quite well, but it is not directly compatible with the flow field outside the vortex, which at most intakes is not axisymmetric or linearly varying along the vortex axis. In this paper, we extract axial stretching and circulation estimates from velocity measurements and a rough potential flow model of the flow approaching the intake and then substitute these values into Burger's model to estimate the vortex characteristics.

\section{Vorticity generation and axial stretching leading to vortex formation}

This section describes common scenarios in which vorticity is generated at the intake or upstream in the intake channel. If the vorticity is advected to the proximity of the submerged inlet, the vertical flow acceleration driven by the inlet axially stretches the vorticity and produces a vortex.

Vorticity can be generated in a shear layer, such as in the boundary layer along the intake channel's lateral walls where a moment imbalance is produced by the retarding 
force of the wall on the flow. High velocity flow entering the channel from the river reach can also generate a shear layer between high and low velocity flow, producing vortices. Vorticity can also be generated by flow separation. If the channel narrows or widens too quickly, recirculating flow may develop in the low pressure region of the wake or in the stagnation zone (Quick, 1962; Gulliver et al., 1986). Similarly, the piers used to hold trash racks across intake openings can obstruct the flow and produce vortices in their wake (see Fig. 2a) (Jiming et al., 2000). Piers often generate problematic vortices because they are located directly adjacent to the intake opening and span the full depth of the intake, so they strengthen the vortices along their whole length.

Vorticity generated a considerable distance upstream may be advected with the flow as mild, harmless vortices until they reach a point above the intake opening where they becomes concentrated into strong and problematic ones. For example, vortices may be generated at the point where flow is diverted laterally from a river into the intake channel (see Fig. 2b). Alternatively, flow may enter the intake channel with a lateral velocity at the free surface, creating a helical flow pattern across the channel cross-section whose lateral velocity component could initiate or strengthen vortices at the piers (see Fig. 3).

At some intakes, vortices may occur only under very specific and rare circumstances. In multi-turbine installations for example, vortices may occur when one or more turbines is not in full operation, producing skewed flow towards the inlets of those turbines that are. Less predictable conditions can include wind, non-uniform ice buildup or floating debris that can modify the flow pattern to produce vortices. Finally, turbulence in the river flow can play a significant role in either providing the seeds for problematic vortices or in breaking them down before they become strong enough to cause harm. Turbulence can 
initiate vortex breakdown by exciting instabilities inherent to the vortex or by stripping vorticity from the primary vortex through the action of secondary ones. Unfortunately turbulence is complex and difficult to characterize so it is difficult to document how it interacts with coherent vortices in any given situation.

\section{Assessing vortex risk}

During the design phase of an intake, engineers use different methods to evaluate the risk that free surface vortices will form over the proposed range of operating conditions. If there appears to be a significant risk, they will attempt to modify the intake within the technical and economic constraints of the project.

As a starting point, vortex risk can be roughly assessed by mapping the proposed submergence-flow rate combinations onto a graph of past studies of vortex activity in intakes with similar configurations, such as Fig. 4, adapted from Fig. 3 of Gulliver et al. (1986). $s$ is the distance from the intake to the free surface, and $d$ is the intake pipe diameter. $g$ is the gravitational acceleration and $U_{i}=4 Q /\left(\pi d^{2}\right)$ is the mean velocity in the intake pipe for the flow rate $Q$. The empty circles in the figure show operating conditions for horizontal intakes where vortices did not form and the filled dots show conditions where problematic vortices did form. The $\times$ 's show the data points from the experiment described in this paper, which was operated at greater relative intake velocities and submergences, which in combination with the piers produce stable vortices and thus ease measurements. The dashed line shows the rough limit between the 'safe' and 'dangerous' conditions, estimated by Gulliver et al. (1986). This approach can give a rough idea of 
vortex risk, but it cannot fully account for particular conditions at a given intake, such as flow asymmetry or geometry, that can significantly influence vortex formation.

More rigorous evaluation of vortex risk is achieved by constructing a physical scale model of the proposed intake and visually inspecting the flow for vortices. Physical models are expensive to build but they allow the engineers to acquire a good sense of how the flow and intake geometry interact to produce flow asymmetries or vortices. Physical models can also be relatively easily modified to evaluate and compare alternative designs. There are however many challenges to evaluating vortex activity in a physical model.

The scaling factor between the prototype and laboratory model for hydropower intakes can range from 1:20 up to as large as 1:200 (Hecker, 1981). At large scaling ratios, the free surface depression produced by vortices in the model can be almost imperceptible. Direct observation of the free surface depression is particularly difficult if the model walls are opaque, in which case the free surface only be observed from above. In this situation, the presence of vortices can be detected by watching for the deformation of reflections on the free surface. The vortices tend to be small compared to the intake, and highly transient in time and place. They sometimes appear and become visible a short distance from the intake and slowly intensify as they approach it. They may then attain a stable intensity and location for several seconds and then dissipate, suddenly or gradually. Once a vortex is detected in a lab-scale physical model, the standard practice is to inject dye into its core to evaluate its coherence and stability or persistence. Engineers usually categorize and record vortices in terms of qualitative characteristics such as the coherence of the dye core and the vortex's ability to entrain floating particles (Hecker, 1987; Walder and Rutschmann, 2007; Mercier et al., 2008; Kiviniemi and Makusa, 2009; Taghvaei et al., 2012). 
The transient nature of the vortices significantly adds to the challenge of documenting and identifying trends in vortex activity. Turbulence can further complicate the task. At relatively low turbulence levels, vortex intensity tends to increase when the flow rate increases. However, when turbulence also increases significantly with increased flow rate, vortex activity may decrease at higher flow rates, suggesting that the turbulence is preventing vortices from forming or intensifying (Padmanabhan and Hecker, 1984; Tastan and Yildırım, 2010).

\section{Quantitative vortex assessment in a physical lab-scale model}

The goal of this paper is to provide tools that will allow engineers to quantitatively assess the characteristics of vortices observed in physical laboratory models using simple and accessible measurement devices or techniques. The processes that link these characteristics to the intake geometry and flow conditions are also quantitatively documented in order to gain greater insight into scaling behavior reported by previous authors. To achieve this, detailed velocity measurements are taken in a simplified physical lab-scale model of an intake, documenting both the approach flow and vortex characteristics such as the characteristic radius $r_{o}$ and bulk circulation $\Gamma_{\infty}$. The measurements are then combined with Burgers's vortex model to produce an analytical model that links the measured vortex characteristics with the approach flow and geometry of the intake. In the experiment, the Froude number $\left(\mathrm{Fr}_{s}\right)$ ranges from 0.4 to 1.1, the Reynolds number $\left(\operatorname{Re}_{s}\right)$ ranges from $8 \times 10^{4}$ to $5.6 \times 10^{5}$, and the Weber number $\left(\mathrm{We}_{s}\right)$ ranges from $800 t o 16.9 \times 10^{3}$, with $\mathrm{Fr}_{s}, \operatorname{Re}_{s}$ and $\mathrm{We}_{s}$ defined in terms of the submergence: $\mathrm{Fr}_{s}=U_{i} /(s g)^{1 / 2}, \operatorname{Re}_{s}=U_{i} s / \nu$ 
and $\mathrm{We}_{s}=\rho U_{i}^{2} s / \sigma$, where $\rho$ and $\nu$ are the water density and kinematic viscosity, respectively, and $\sigma$ is the surface tension coefficient for a clean air-water interface.

The analytical model is first used as a guide for estimating the characteristic radius of the vortex from velocity measurements of the approach flow made with an Acoustic Doppler Velocimeter (ADV), and for roughly measuring the bulk circulation using a relatively simple surface particle-tracking technique. In the following section, the analytical model is used to evaluate how the documented processes scale and thereby influence how vortex characteristics observed in a laboratory scale physical model might scale up to the prototype scale.

\section{Experimental setup}

The physical model in which the measurements are made has a $3.9 \mathrm{~m}$ long channel, a square 1 by $1 \mathrm{~m}$ cross-section and a circular pipe of inner diameter $d=11.5 \mathrm{~cm}$ mounted flush into the downstream wall of the channel, with its axis located $0.14 \mathrm{~m}$ above the channel bed (Fig. 5). The geometry is described in more detail in Suerich-Gulick et al. (2013c). Two tall narrow plates are mounted perpendicular to the downstream wall on each side of the outlet opening. These plates protrude a distance $l_{p}=45 \mathrm{~mm}$ away from the wall and produce a more stable vortex by provoking flow separation; they are spaced $k=15 \mathrm{~cm}$ apart, symmetrically about the pipe axis, and they span the full channel depth. They are referred to as piers in the rest of this paper since they act in the same way as the piers that hold trash racks across the penstock opening at hydropower intakes. Each pier generates a relatively stable vortex pair in its wake: one vortex that starts at the free surface and one that starts from the floor of the tank. Both vortex tails are entrained into the inlet 
pipe, but we focus on the free surface vortices in this paper because these are more likely to cause problems by entraining air or floating debris. In most hydropower intakes, the submerged vortices are minimized or absent because the inlet opening is roughly aligned with the bottom of the channel. Only the right-hand free surface vortex is measured (as seen looking downstream) because of geometrical symmetry. Minor mean asymmetry arises due to asymmetry of the supply pipe and temporal asymmetry arises due to the interaction of the two vortices. A global coordinate system $(X, Y, Z)$ with corresponding velocities $\left(U_{X}, U_{Y}, U_{Z}\right)$ is defined to refer to the geometry and flow outside the vortex. Its origin is located at the free surface, half-way across the downstream wall of the channel. $Z$ points down towards the bed and $X$ points downstream. A local coordinate system $(r, \theta, z)$ with corresponding velocities $\left(V_{r}, V_{\theta}, V_{z}\right)$ is defined at the vortex axis with $z$ pointing down from the free surface.

\section{Estimating the characteristic radius from the approach flow}

The characteristic radius $r_{o}$ is a key determinant of vortex intensity that is also quite difficult to measure directly. However it is possible to estimate $r_{o}$ from the vertical gradient of the approach flow velocity $\partial|U| / \partial Z$ directly in front of the inlet. This section describes how mean flow velocity measurements made in the simplified intake lab model can be used to calculate $r_{o}$ (Suerich-Gulick et al., 2013c). The calculations can be adapted to other geometries and configurations.

The magnitude of velocity $|U|$ for the approach flow is measured using a Sontek MicroADV along a vertical line located at the channel centerline $(Y=0), \triangle X=5.5 \mathrm{~cm}$ upstream from the inlet pipe. The ADV sampling volume is located $55 \pm 2 \mathrm{~mm}$ away 
from the probe tip and is roughly cylindrical, $1 \mathrm{~cm}$ in diameter and length. The velocity is recorded at $30 \mathrm{~Hz}$ and averaged over 2 minutes for each measurement location, with no filtering. The correlation values are above 0.7 for all but a few isolated measurement locations, whose average measured velocities are consistent with those of the neighboring locations.

It is found that the bulk flow between the piers in the upper portion of the channel behaves like potential flow drawn into a horizontal line sink along $Y$ located at the upper edge ( $Z=s$ of the inlet opening (Yıldırım et al., 2000). (See Fig. 6(a) for a schematic section of the flow.) The non-dimensional velocity $|U| / U_{i}$ thus collapses onto a single curve for all eight flow conditions studied, which makes it possible in the next section to establish relations for $r_{o}$ and $\Gamma_{\infty}$ in terms of the mean inlet velocity $U_{i}$ and relative submergence $s / d$ :

$$
\frac{|U|_{\text {fit }}(\eta)}{U_{i}}=\frac{\mathrm{c}_{1} d}{4 k}\left(\frac{d}{\eta}-\mathrm{c}_{2}\right)
$$

where $|U|=\left(U_{X}^{2}+U_{Y}^{2}+U_{Z}^{2}\right)^{1 / 2}$, and $\eta$ is the total distance from the top of the inlet opening to each measurement point (as shown in Fig. $6 a$ ), so that $\eta=\sqrt{(s-Z)^{2}+(\triangle X)^{2}} \cdot Q$ is the flow rate through the intake pipe (units $\mathrm{m}^{3} / \mathrm{s}$ ). The non-dimensional coefficients $\mathrm{c}_{1}=0.8$ and $\mathrm{c}_{2}=0.28$ are selected to produce the best fit. This best fit curve $|U|_{\text {fit }} / U_{i}$ is plotted as a dashed line in Fig. $6(b)$. The line for $|U|$ (outside the vortex) is truncated at the value of $\eta / d$ where the free surface is located for the appropriate submergence level $s / d$.

The deepest submergence level $s / d=3.4$ is selected as the upper limit of operating conditions for study in the experiment because vortex activity becomes much more sporadic above that level. This transition point in vortex activity occurs when the vertical profile of approach flow velocity approaches zero at the free surface, indicating that a 
small degree of recirculation about the horizontal axis starts to occur where the free surface meets the intake wall. Since there appears to be a qualitative shift in flow structure at the $s / d=3.4$ submergence, some caution should be used in applying trends observed at lower submergences to predict behavior at submergences equal to or greater than this.

Once the velocity profile of the approach flow has been established, it can be used to estimate the characteristic radius $r_{o}$. The measurements show that the mean slope of the axial velocity profile $V_{z}(z)$ inside the vortex is driven by the velocity profile $|U|(Z)$ outside the vortex over the same vertical section of flow (see Fig. 6b). For a majority of the operating conditions, $V_{z}$ follows a linear profile in $\eta$ over a significant portion of the upper flow instead of growing as $\eta^{-1}$ as does $|U|$. The pressure gradient within the vortex possibly acts to equalize the axial $V_{z}$ gradient so that it tends towards the linear profile $V_{z}=a z$, with a slope $a$ that is roughly equal to the mean of $|U|$ over the same section. Closer to the inlet pipe, the axial gradient or absolute value of $|U|$ must be too strong for the linearization to occur.

The distance over which the linear $V_{z}$ profile forms varies with operating conditions in a way that is difficult to predict from the available data, so instead of predicting a single value for the axial slope $a$, a range of values is estimated, within which it should fall. If no linearization occurs, then the axial velocity gradient at the free surface inside the vortex should roughly match $|U|$ outside the vortex. If linearization occurs over a proportion $\beta$ of the submergence $s$, then it is estimated that $V_{z}(z)$ will follow a straight line from $V_{z}=|U|_{\eta \approx s}$ at the free surface to $V_{z}=|U|_{\eta \approx(1-\beta s)}$ a distance of roughly $\beta s$ below the 
free surface, closer to the inlet pipe. The resulting gradient is

$$
a_{\mathrm{est}}=\frac{\mathrm{c}_{1} U_{i} d^{2}}{4 k s^{2}(1-\beta)},
$$

where $0<\beta<0.85$, and $\beta=0$ corresponds to no linearization. This estimate can be compared to that obtained directly from the vertical gradient of the measured $|U|$ profiles at the free surface (for $\beta=0$ ) or over the top portion of the flow (for $\beta \neq 0$ ). Once the axial velocity gradient has been estimated, the characteristic radius $r_{o}$ can be calculated using Eq. (2):

$$
r_{o, \mathrm{est}}=\frac{4 s}{d}\left(\frac{\nu k(1-\beta)}{\mathrm{c}_{1} U_{i}}\right)^{1 / 2}
$$

\section{Bulk circulation}

The bulk circulation $\Gamma_{\infty}$ can be measured in the physical model at the free surface using surface particle tracking or it can be roughly estimated from the measured magnitude of the approach velocity at the free surface.

Surface particle tracking is achieved by placing floating particles on the free surface near the vortex and watching how fast they rotate around the vortex once they are entrained into its domain of influence. If the velocities are very high, it may be necessary to film the particles or measure the angle spanned by particle streaks on still images of their trajectory. If a particle completes $N$ full rotations every second around a circular path of radius $r_{i}$ about of the vortex, then the circulation at that radius $r_{i}$ is roughly $\Gamma\left(r_{i}\right)=4 \pi^{2} r_{i}^{2} N$. Ideally, several measurements of $\Gamma_{\infty}$ should be taken for a given operating condition since significant variation in the circulation for a given vortex and from vortex to vortex is com- 
mon.

In order to gain insight into scaling behavior, we want to relate the observed circulation to the operating condition parameters $U_{i}$ and $s / d$. Normally, the circulation should roughly scale with the product of the approach velocity at the free surface $|U|_{\mathrm{fs}}$ near where the vortices form and a length scale $l$ that determines the zone in which the circulation may establish itself. These quantities are fairly easy to determine for the intake model considered here, because the vortices are generated by an obvious mechanism (separation off the pier tip) and in a clearly defined zone (the space between the piers). Measurements of the bulk circulation around the vortex reveal that it scales quite well with $|U|_{\mathrm{fs}} \pi l_{p}$, where $l_{p}=1.2 \mathrm{~cm}$ is the length of the pier. Using Eq. (3) with $\eta=s$ for $|U|_{\mathrm{fs}}$, this relation yields

$$
\Gamma_{\infty, \mathrm{est}} \approx \frac{\mathrm{c}_{3} \mathrm{c}_{1} d U_{i} \pi l_{p}}{4 k}\left(\mathrm{c}_{4} d / s-\mathrm{c}_{2}\right)
$$

where the coefficients $c_{3}=0.33$ with $c_{4}=1.0$ fit the lower limit of the measured values for $\Gamma_{\infty}$, and the upper limit of the measured values is given by $c_{3}=0.33$ with $c_{4}=1.8$.

\section{Free surface depression}

The pressure drop due to centripetal acceleration causes the water level to drop in the vortex's center. The radial profile of the free surface depression for a given azimuthal velocity profile $V_{\theta}(r)$ can be computed by the following relation with sufficient accuracy if the axial and radial velocities near the free surface are small compared to $V_{\theta}$ :

$$
h(r)=\int_{\infty}^{r}\left(\frac{V_{\theta}\left(r^{\prime}\right)^{2}}{g r^{\prime}}-l_{\sigma}^{2} \kappa\left(r^{\prime}\right)\right) d r^{\prime}
$$


where $h(r)$ is the vertical distance from the undeformed free surface level to that of the deformed free surface. $\kappa(r)$ is the local mean curvature of the air-water interface, $l_{\sigma}=$ $\sqrt{\sigma /(\rho g)}$ is the characteristic length of the air-water interface, and $\sigma$ is the surface tension coefficient (Andersen et al., 2006). A constant value of $l_{\sigma}=2.73 \mathrm{~mm}$ is used here, which corresponds to a clean air-water interface at $15^{\circ} \mathrm{C}$. The first term on the right-hand side represents the centripetal acceleration that reduces the pressure inside the vortex, pulling the free surface interface downward. The second term represents the upward force exerted by surface tension. The mean free surface curvature $\kappa(r)$ is given by

$$
\kappa(r)=-\frac{1}{2}\left[\frac{h_{r}}{r\left[1+\left(h_{r}\right)^{2}\right]^{1 / 2}}+\frac{h_{r r}}{\left[1+\left(h_{r}\right)^{2}\right]^{3 / 2}}\right]
$$

where $h_{r}$ and $h_{r r}$ are the first and second derivatives of $h$ with respect to $r$ respectively. The first term on the right is the curvature about the horizontal axis and the second is the curvature about the vortex's (vertical) axis of rotation.

Two quantities are important to evaluate if a vortex will cause operation problems: the overall shape of the free surface depression and its maximum depth $h_{0} \equiv h(0)$, which will be referred to hereafter as the tip depth. The nominal slope $h_{0} / r_{o}$ of the free surface depression is used hear as as a representative quantity of the vortex shape. The shape of the depression impacts both surface tension effects and the detachment of air bubbles down from the tip of the depression (Andersen et al., 2006). To compute the slope and tip depth, the expressions for $\Gamma_{\infty}$ and $r_{o}$ (Eqs. 6 and 5 respectively) are substituted into Burgers's profile for $V_{\theta}(r)$ (Eq. 1), which is substituted into the equation for the free surface profile (Eq. 7). Since the resulting equation is non-linear in $h(r)$, an approximate solution for the tip depth is computed. First, the first term in the integral of equation (Eq. 7) is integrated 
from $r=\infty$ to $r=0$, which gives the relative tip depth $h_{\mathrm{n}, 0} / d$ without surface tension effects:

$$
h_{\mathrm{n}}^{\prime} \equiv \frac{h_{\mathrm{n}, 0}}{d}=\frac{0.17 \Gamma_{\infty}^{2}}{g r_{o}^{2}}=\frac{\mathrm{c}_{5} \mathrm{c}_{3}^{2} \mathrm{c}_{1}^{3}}{(1-\beta)}\left(\frac{U_{i}^{3}}{g \nu}\right)\left(\frac{d}{k}\right)^{3}\left(\frac{l_{p}}{s}\right)^{2}\left(\mathrm{c}_{4} d / s-\mathrm{c}_{2}\right)^{2},
$$

where $c_{5}=0.17 / 16^{2}=6.6 \times 10^{-4}$ is determined by the integration, and $U_{i}^{3} /(g \nu)=$ $\operatorname{Re}_{s} \mathrm{Fr}_{s}^{2}$.

The nominal slope $h_{\mathrm{n}, 0} / r_{o}$ is determined by Eqs. (9) and (5):

$$
\frac{h_{\mathrm{n}, 0}}{r_{o}}=\frac{\mathrm{c}_{5} \mathrm{c}_{3}^{2} \mathrm{c}_{1}^{7 / 2} d^{5} l_{p}^{2}}{(1-\beta)^{3 / 2}(s k)^{7 / 2}}\left(\frac{U_{i}^{3}}{g \nu}\right)\left(\mathrm{c}_{4} d / s-\mathrm{c}_{2}\right)^{2} .
$$

$r_{o}$ and $h_{\mathrm{n}, 0} / r_{o}$ are then used to obtain the surface tension correction factor $f_{\sigma}=\Delta h / h_{\mathrm{n}, 0}$ from Fig. 7(a), where $\Delta h$ is the difference between the tip depth with and without surface tension. The tip depth with surface tension $h_{\sigma, 0}$ is given by: $h_{\sigma, 0}=h_{\mathrm{n}, 0}\left(1-f_{\sigma}\right)$. Surface tension changes the shape of the depression as well as its tip depth, but its effect on shape is generally small enough that it does not change the magnitude of the relative surface tension effect to a significant degree.

Figure $7(a)$ is a compilation of the results of finite difference simulations of the effect of surface tension on the free surface depression produced by a Burgers's vortex for a wide range of vortex scales $r_{o} / l_{\sigma}$ and shapes $r_{o} / h_{\mathrm{n}, 0}$ (Suerich-Gulick et al., 2013b). It reveals that the relative surface tension effect is significant at scales comparable to $l_{\sigma}$, but negligible for $r_{o} / l_{\sigma}>5$. The effect is also much greater for dimple-shaped depressions $\left(h_{\mathrm{n}, 0} / r_{o} \lesssim 1\right)$ than for funnel-shaped depressions $\left(h_{\mathrm{n}, 0} / r_{o} \gtrsim 5\right)$, which are shown in Fig. 7(b). This result is relevant because many past laboratory studies have focused on 
scale effects in air core vortices (a subset of funnel-type vortices) whereas the vortices observed in physical scale models of large hydropower projects tend to produce dimpleshaped depressions.

In summary, the vortex characteristics in the lab-scale model can be estimated from the intake velocity $U_{i}$ and relative submergence $s / d$ using the relations developed above: $r_{o}$ can be estimated using Eq. (5), $\Gamma_{\infty}$ from Eq. (6), $h_{\mathrm{n}, 0} / d$ from Eq. (9), and the shape $h_{\mathrm{n}, 0} / r_{o}$ from Eq. (10). In order to adapt this analytical model for a slightly different intake, one would first need to measure the mean velocity profile of $|U|(Z)$ directly in front of the intake, over a range of operating conditions. The bulk circulation $\Gamma_{\infty}$ of the vortices could be roughly measured using surface PTV. These data would then be used to adjust coefficients $c_{1}, c_{2}$ and $c_{3}$ in Eqs. (3) and (6), assuming the flow structure and circulation-generating mechanism are essentially the same as in this experiment. The range of $r_{o}$ values would be estimated using Eq. (2) with the limiting values $\beta=0.15$ and 0.85 . Stronger vortices at shallower submergences would probably have a larger value of $\beta$. If the form of Eqs. (3) and (6) has not been modified, the tip depth $h_{\mathrm{n}, 0}$ and the nominal depression slope $h_{\mathrm{n}, 0} / r_{o}$ can be computed directly from Eqs. (9), and (10), respectively. $c_{5}$ is not empirically adjusted and so remains constant. The relative surface tension effect $\Delta h / h_{\mathrm{n}, 0}$ can be read off the graph in Fig. 7(a).

Compared to the current experiment, hydropower intakes often have a larger and/or rectangular opening, followed by a smooth transition to the circular penstock. A larger opening produces a lower mean intake velocity $U_{i}$, which could in turn reduce the axial stretching of the vortex for a same submergence. An equivalent hydraulic diameter $d_{\mathrm{h}}$ of the opening should be used in this case. 
Eq. (2) could also be used to estimate the characteristic radius from the solution of a rough computational fluid dynamics (CFD) simulation where the vortex is too diffuse, either due to a coarse mesh or simplified turbulence model producing excessive eddy diffusivity. In this case, the simulated $V_{z}(z)$ profile inside the vortex would likely not be as linear as in the experiment, so $a$ can be estimated from the velocity profile $|U|(Z)$ directly upstream from the intake (Suerich-Gulick et al., 2013a):

$$
a_{\mathrm{est}, C F D}=\frac{\left\{U_{Z}(\beta s)-U_{Z}(0)\right\}}{\beta s} .
$$

Values of $\beta$ ranging from 0.15 to 0.85 as observed in the experiment (Suerich-Gulick et al., 2013c) would give a range of values for $a_{\text {est,CFD }}$, which could be substituted into Eq. 2 to obtain a corresponding range of $r_{o}$. The peak azimuthal velocity $V_{\theta, \max }=0.1 \Gamma_{\infty} / r_{o}$ and the tip depth $h_{\mathrm{n}, 0}$ (the depression at $r=0$ neglecting surface tension effects) can then be computed using $h_{\mathrm{n}, 0}=0.17 \Gamma_{\infty}^{2} /\left(\pi^{2} r_{o}^{2} g\right)$, with $\Gamma_{\infty}$ extracted directly from the simulation by fitting Eq. (1) to the $V_{\theta}(r)$ profile.

\section{Scale effects}

Empirical data about how free surface vortices affect turbine performance is limited, and the necessary conditions that would allow observations of vortex characteristics made in a laboratory-scale model to be directly translated to the prototype scale are unclear. This double uncertainty makes it quite difficult for engineers to determine if a vortex is truly 'problematic' when it is observed in a lab-scale physical model. The second source of uncertainty is addressed in this section. 
Past researchers have focused primarily on the magnitude of the free surface depression when trying to evaluate the effect of scale on vortex 'strength', because the threat of air entrainment by the vortices is the least ambiguous (Daggett and Keulegan, 1974; Anwar, 1983; Möller et al., 2012). Daggett and Keulegan (1974) and Anwar (1983) found that the critical parameters in the laboratory model became less sensitive to the Reynolds number for $\operatorname{Re}_{s} \gtrsim 4 \times 10^{4}$ or $1 \times 10^{5}$, respectively. Similarly, Anwar (1983) found that surface tension effects were less sensitive to the Weber number for $\mathrm{We}_{s}>1.5 \times 10^{4}$ or $4 \times 10^{4}$ if the vortex was a dimple or an air core, respectively.

The analytical model described in the previous sections is used to examine how the scaling behavior of the experimentally documented processes should influence the translation of vortex characteristics observed in a laboratory-scale model to the prototype scale. It is assumed that the geometry of the lab- and prototype-scale intakes is identical, with a scaling factor $\alpha$, meaning for example that the ratio of the prototype to model diameters $d_{P} / d_{M}=\alpha$. It is also assumed that the lab-scale model is operated according to Froude similitude $\operatorname{Fr}_{M}=\mathrm{Fr}_{P}$, which produces a ratio of the prototype and lab-scale Reynolds numbers $\operatorname{Re}_{P} / \operatorname{Re}_{M}=\alpha^{3 / 2}$. If the flow structure outside and inside the vortex follows the same shape at both scales so that $\beta_{P}=\beta_{M}$ and the coefficients in Eq. (9) are identical at both scales, the ratio of the relative tip depths predicted by Eq. (9) is then $h_{P}^{\prime} / h_{M}^{\prime}=\alpha^{3 / 2}$, and the ratio of the depression slopes predicted by Eq. (10) is $h_{P}^{\prime} / h_{M}^{\prime}=\alpha^{3 / 4}$.

This indicates that the relative tip depth in a prototype intake would be 460 times as large as that in the laboratory model if the latter was constructed at a 1:60 scale, whereas the ratio of the depression slopes would be 22 . This contradicts the results of many previous authors in which sensitivity to $\operatorname{Re}_{s}$ appears to decrease asymptotically at larger scales 
and values of $\operatorname{Re}_{s}$, suggesting that a key process is missing from the analytical model. The most promising explanation for this asymptotic trend is that radial turbulent diffusion in the vortex may increase at larger scales instead of being entirely suppressed as it is in the current experiment. In this case, the effective diffusivity due to turbulence could become sufficiently important at high Reynolds numbers that molecular viscosity would become insignificant in comparison (Odgaard, 1986). The impact of such a process can be tested analytically by replacing $\nu$ in Eqs. (2) and (9) by an effective viscosity $\nu_{\text {eff }}=\nu+\nu_{\mathrm{T}}$, with $\nu_{\mathrm{T}}=\chi \Gamma_{\infty}$ and the dimensionless coefficient $\chi=6 \times 10^{-5}$ as sugested by (Odgaard, 1986). As shown in Fig. 8, the relative free surface tip depth $h_{\mathrm{n}, 0} / d$ predicted by Eq. 9 becomes asymptotically independent of $\operatorname{Re}_{s}$ at values ranging from $10^{5}$ to $10^{7}$ as a result. The transition point depends on the relative submergence $s / d$, as shown in Fig. 8. In comparison, $h_{\mathrm{n}, 0} / d$ increases linearly with $\operatorname{Re}_{d}$ if $\nu_{\mathrm{eff}}=\nu$. It is assumed here that $\beta$ and the flow structure outside the vortex are independent of $\operatorname{Re}_{s}$; values $\beta=0.85$ and $c_{4}=1$ are used to generate the curves in the graph. This explanation is sufficiently promising to warrant more work to evaluate the effective viscosity controlling the characteristic radius $r_{o}$ at different $\operatorname{Re}_{s}$ values. Other processes that could generate a decreased sensitivity to $\operatorname{Re}_{s}$ at higher $\operatorname{Re}_{s}$ values include a change in the structure of the intake flow due to decreased relative viscous effects, or a change in the axial stretching inside the vortex at larger scales (producing different $\beta$ values). In addition, greater turbulence at the prototype scale might prevent certain vortices from forming that occur in the laboratory-scale intake where turbulence is weaker. 


\section{Summary and conclusions}

Using measurements in a laboratory-scale intake model and Burgers's vortex model, an analytical model was developed that relates key vortex parameters such as the characteristic radius, bulk circulation and free surface depression depth and shape to the intake geometry and approach flow conditions. The analytical model helps to understand how different processes affect the scaling behavior of the vortex characteristics. If turbulence is entirely suppressed in the vortex, the analytical model predicts that the ratio of the prototype- to lab-scale relative tip depths should be proportional to $\alpha^{3 / 2}$ and the ratio of the depression slopes should be proportional to $\alpha^{3 / 4}$. If turbulent diffusivity in the vortex increases at greater $\operatorname{Re}_{s}$ values, the model predicts that the relative tip depth will become independent of $\operatorname{Re}_{s}$ beyond a certain limit value of $\operatorname{Re}_{s}$ that varies with relative submergence.

Future work is required to examine what determines the degree of linearization of the axial velocity profile inside the vortex. Greater understanding of the scaling behavior of the processes could be achieved by studying the same set of geometry and flow conditions at different model sizes. Significantly larger models would be required to clarify the impact of turbulence on vortex stability and on the effective diffusivity that controls the vortex characteristic radius.

We would like to thank David Morissette, Pierre Tadeo, Tristan Aubel, Maryse Page, Anne-Marie Giroux and Sébastien Houde for their advice and contributions. F. S.-G. was supported by the National Science and Engineering Research Council, the Fonds Québécois pour les Sciences et les Technologies, and the Hydro-Québec research center. 


\section{REFERENCES}

Andersen, A., Bohr, T., Stenum, B., Rasmussen, J. J., and Lautrup, B. (2006). "The bathtub vortex in a rotating container." J. Fluid Mech., 556, 121-146.

Anwar, H. O. (1983). "The non-dimensional parameters of free-surface vortices measured for horizontal and vertically inverted intakes." Houille Blanche, 1, 11-25.

Burgers, J. M. (1948). "A mathematical model illustrating the theory of turbulence." $A d v$. in Appl. Mech., 1, 171-199.

Daggett, L. L. and Keulegan, G. H. (1974). "Similitude in free-surface vortex formations." J. Hydraul. Div. ASCE, 100(HY11), 1565-1581.

Gulliver, J. S., Rindels, A. J., and Lindblom, K. C. (1986). "Designing intakes to avoid free-surface vortices.” Int. Water Power Dam Constr., 38(9), 24-28.

Hecker, G. (1981). "Model-prototype comparison of free surface vortices." J. Hydraul. Div. ASCE, 107(HY10), 1243-1259.

Hecker, G. E. (1987). "Fundamentals of vortex intake flow." Swirling flow problems at intakes, J. Knauss, ed., A.A. Balkema, Rotterdam, 13-38.

Jiming, M., Yuanbo, L., and Jitang, H. (2000). "Minimum submergence before doubleentrance pressure intakes." J. Hydraul. Eng.-ASCE, 126(8), 628-631.

Kiviniemi, O. and Makusa, G. (2009). "A scale model investigation of free surface vortex with particle tracking velocimetry." M.S. thesis, University of Oulu, Finland, and Luleå University of Technology, Oulu, Finland and Luleå, Sweden. 
Mercier, A., Denault, C., and Villeneuve, M. (2008). "Processus de conception du canal d'amenée de la centrale de la Sarcelle." Canadian Dam Assoc. 2008 Annual Conf., Winnipeg, Canada.

Möller, G., Detert, M., and Boes, R. (2012). "Air entrainment due to vortices - state-ofthe-art.” Proc. 2nd IAHR Europe Cong., paper B16.

Odgaard, A. J. (1986). "Free-surface air core vortex." J. Hydraul. Eng.-ASCE, 112(7), $610-620$.

Padmanabhan, M. and Hecker, G. E. (1984). "Scale effects in pump sump models." $J$. Hydraul. Eng.-ASCE, 110(HY11), 1540-1556.

Quick, M. (1962). "Scale relationships between geometrically similar free spiral vortices, Pt. 2." Civ. Eng. Public Works Rev., 57, 1319-1320.

Saffman, P. S. (1992). Vortex Dynamics. Cambridge University Press, Cambridge.

Suerich-Gulick, F., Gaskin, S. J., Parkinson, E., and Villeneuve, M. (2013a). “Computational fluid dynamics modelling strategies for predicting free surface vortices at hydropower intakes." Manuscript.

Suerich-Gulick, F., Gaskin, S. J., Villeneuve, M., and Parkinson, E. (2013b). "Free surface intake vortices: Scale effects due to surface tension and viscosity." Manuscript.

Suerich-Gulick, F., Gaskin, S. J., Villeneuve, M., and Parkinson, E. (2013c). "Free surface intake vortices: Theoretical model and measurements." Manuscript. 
Taghvaei, S. M., Roshan, R., Safavi, K., and Sarkardeh, H. (2012). "Anti-vortex structures at hydropower dams." Int. J. Phys. Sci., 7(28), 5069-5077.

Tastan, K. and Yıldırım, N. (2010). "Effects of dimensionless parameters on air-entraining vortices.” J. Hydraul. Res., 48(1), 57-64.

Walder, S. and Rutschmann, P. (2007). "Hybrid modeling of an intake for hydraulic optimization." Proc. 32nd IAHR Congress, Venice,Italy.

Y1ldırım, N., Kocabaş, F., and Gülcan, S. (2000). "Flow-boundary effects on critical submergence of intake pipe." J. Hydraul. Eng.-ASCE, 126(4), 288-297. 


\section{List of Figures}

1 (a) Plan and (b) section views of sample run-of-river hydropower installa-

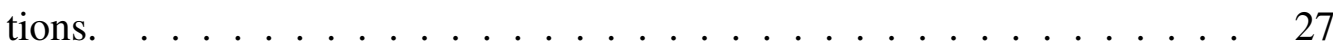

2 (a) Generation of vortices in the wake of piers. (b) Upstream generation and advection of vortices in a wake produced at the junction of the river reach and intake channel. . . . . . . . . . . . . . 28

3 Large-scale helical flow pattern driven by river flow at the the intake channel entrance. . . . . . . . . . . . . . . . . 29

$4 \quad$ Vortex risk vs submergence and intake Froude number. Data from Gulliver

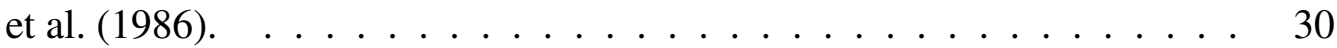

5 (a) Vertical section and (b) isometric views of the simplified laboratory model $($ dimensions in $\mathrm{cm}) . \ldots \ldots 31$

6 (a) Side-view section of the laboratory intake model, with $|\boldsymbol{U}|$ indicated by shading. - - - : the ADV measurement axis. (b) Velocity measured inside and outside the vortex $\left(\boldsymbol{V}_{\boldsymbol{z}}\right.$ and $|\boldsymbol{U}|$, respectively $) \ldots \ldots 32$

7 Relative surface tension effect $\Delta h / h_{\mathrm{n}, 0}$ as a function of the scale and shape of the depression $(a)$. The different depression shapes $(b)$ : dimple, transition and funnel $\left(\boldsymbol{h}_{\mathrm{n}, 0} / \boldsymbol{r}_{\boldsymbol{o}}=0.5,2.5\right.$ and 6.8 , respectively $) . \ldots 33$

$8 \quad$ Scaling of $h_{\mathrm{n}, 0} / d$ for different values of $\nu_{\mathrm{eff}}$ and $s / d \ldots \ldots \ldots$ 

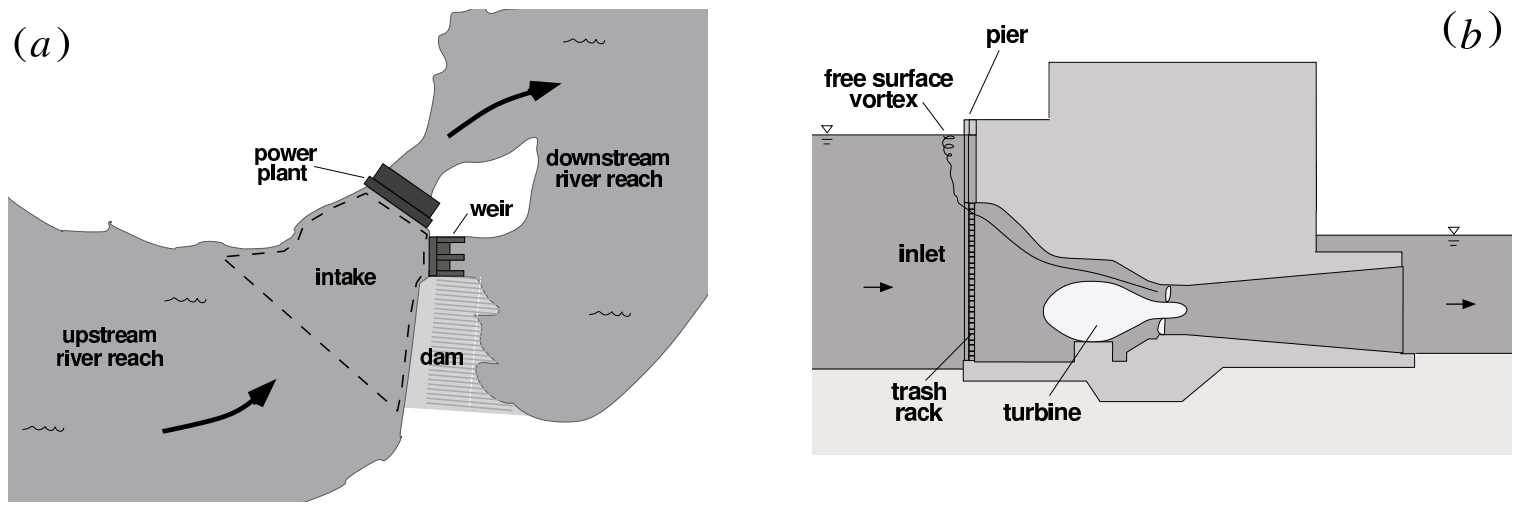

FIG. 1. (a) Plan and (b) section views of sample run-of-river hydropower installations. 

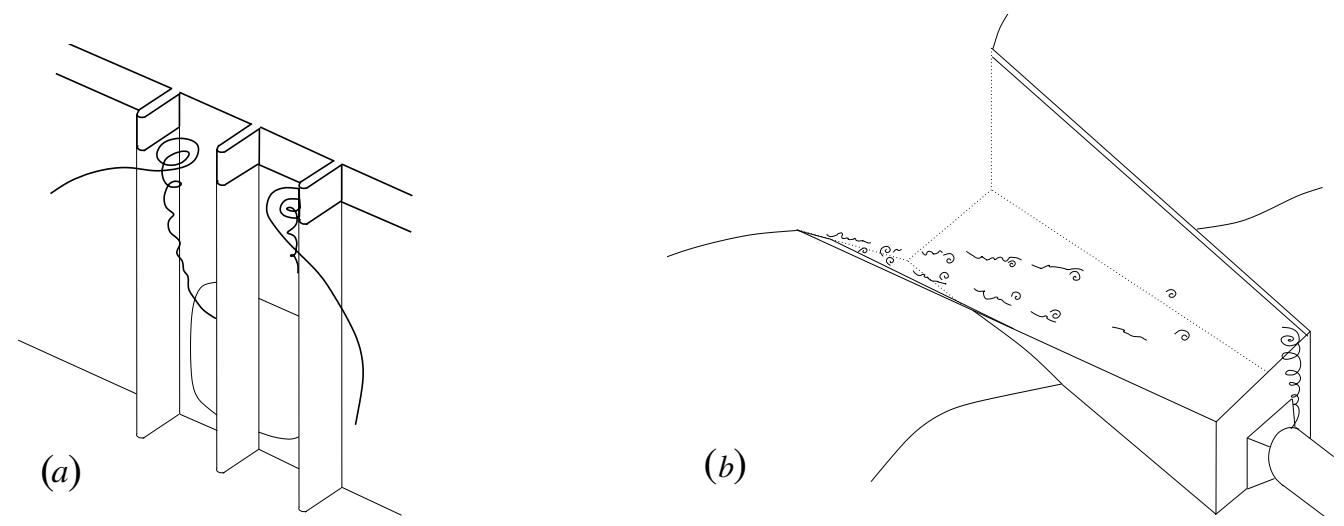

FIG. 2. (a) Generation of vortices in the wake of piers. (b) Upstream generation and advection of vortices in a wake produced at the junction of the river reach and intake channel. 


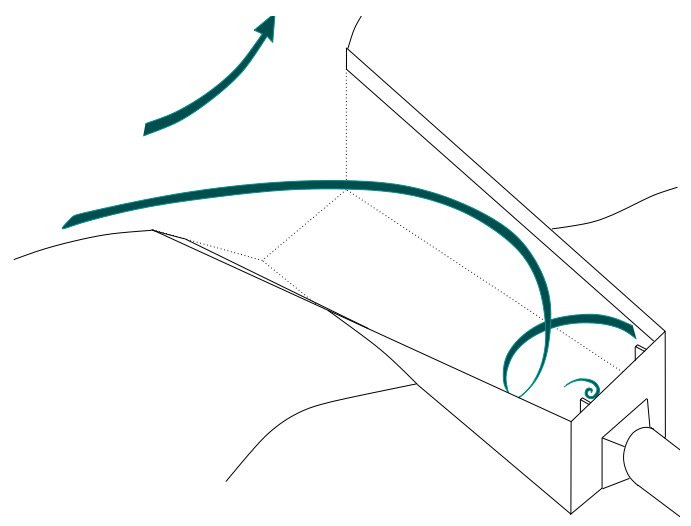

FIG. 3. Large-scale helical flow pattern driven by river flow at the the intake channel entrance. 


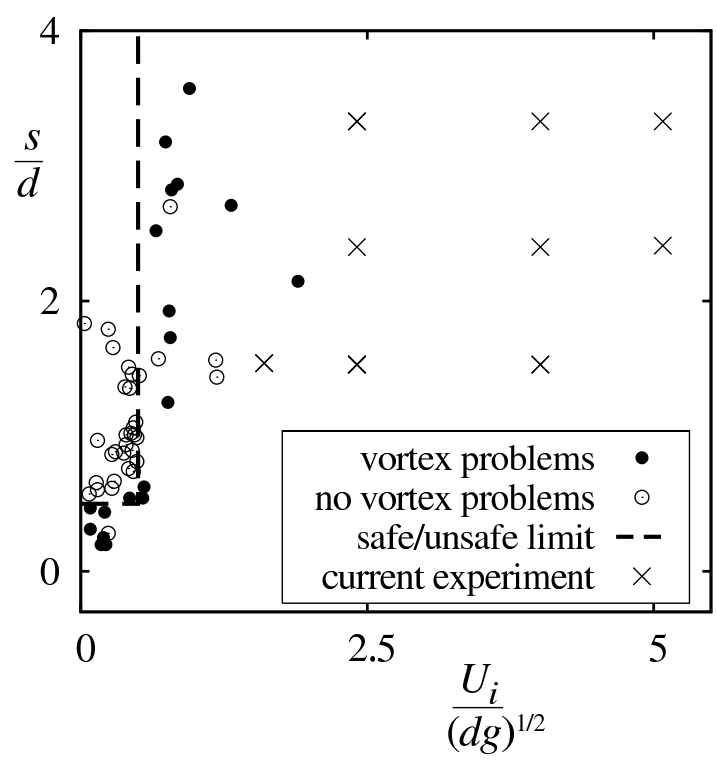

FIG. 4. Vortex risk vs submergence and intake Froude number. Data from Gulliver et al. (1986) and current study. 

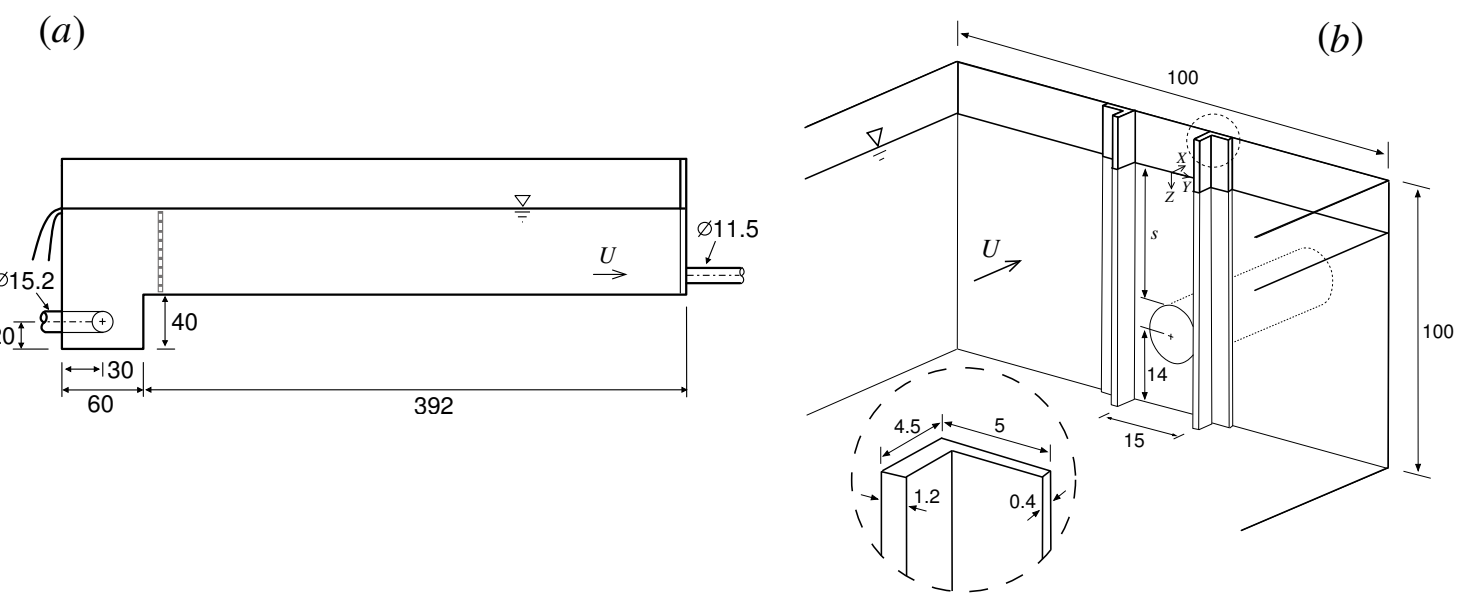

FIG. 5. (a) Vertical section and (b) isometric views of the simplified laboratory model (dimensions in $\mathrm{cm}$ ). 

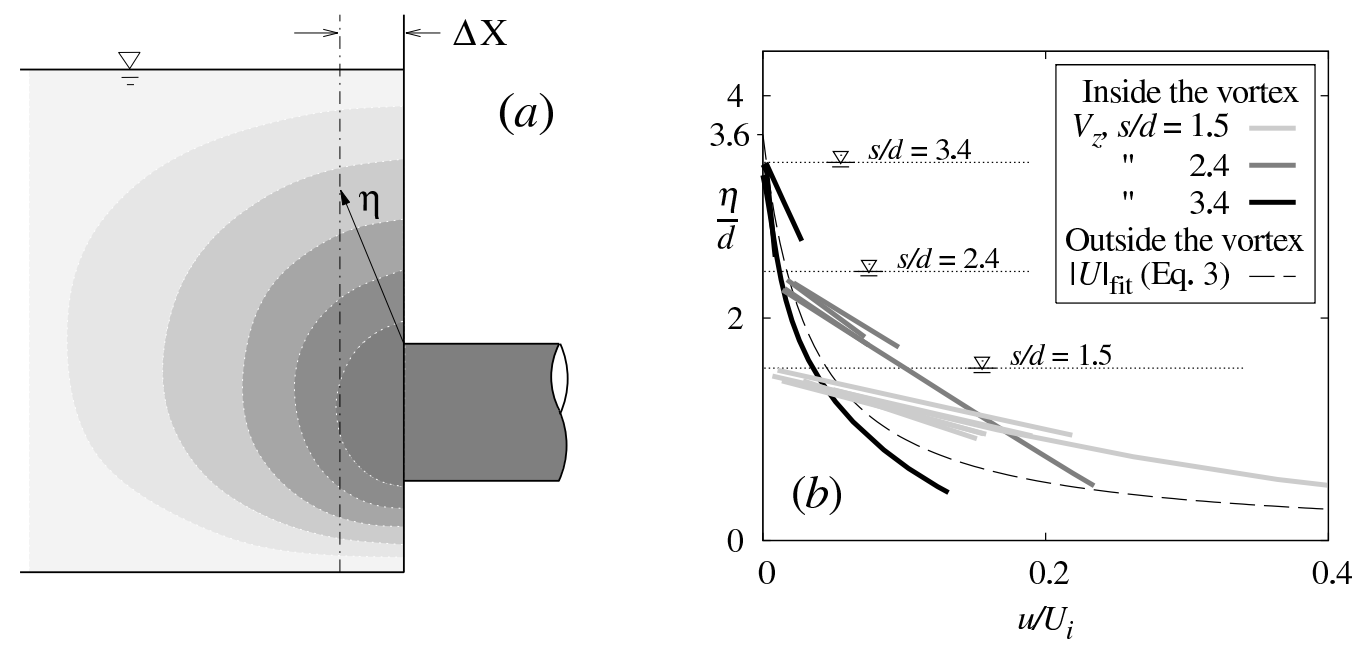

FIG. 6. (a) Side-view section of the laboratory intake model, with $|U|$ indicated by shading. - - - : the ADV measurement axis. (b) Velocity measured inside and outside the vortex $\left(V_{z}\right.$ and $|U|$, respectively). 

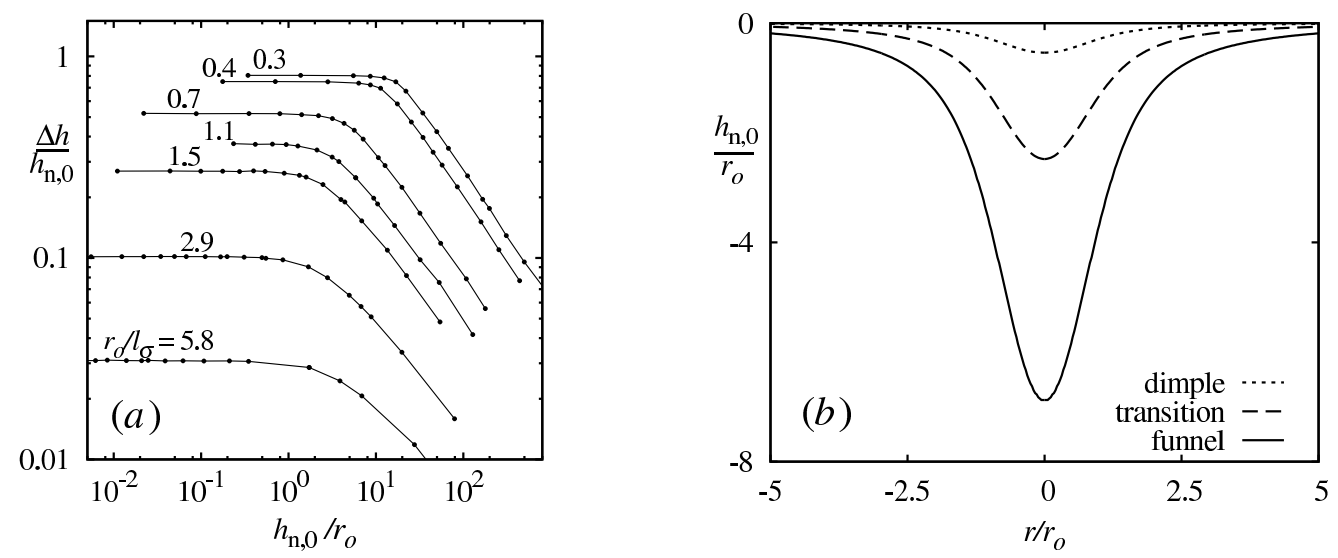

FIG. 7. Relative surface tension effect $\Delta h / h_{\mathrm{n}, 0}$ as a function of the scale and shape of the depression (a). The different depression shapes (b): dimple, transition and funnel $\left(h_{\mathrm{n}, 0} / r_{o}=0.5,2.5\right.$ and 6.8 , respectively). 


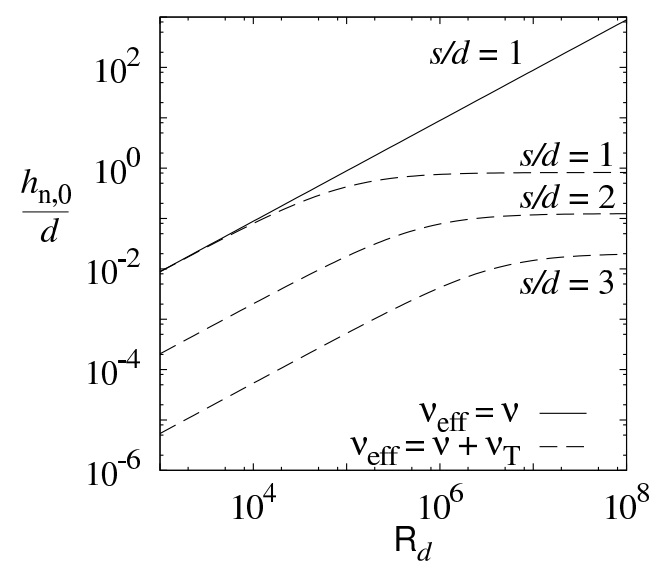

FIG. 8. Scaling of $h_{\mathrm{n}, 0} / d$ for different values of $\nu_{\mathrm{eff}}$ and $s / d$. 